\title{
SYSTEM OF SYSTEMS - A HOLISTIC APPROACH FOR TELEMEDICINE
}

\author{
Viorel Petcu ${ }^{1}$ and Adrian Petrescu ${ }^{2}$ \\ ${ }^{1}$ Research and Technology Department, UTI Systems SA Soseaua Oltenitei 107-111, \\ 041303, Bucharest, Romania, viorel.petcueuti.ro \\ ${ }^{2}$ Computer Science Department Faculty of Automatics, Politehnica University Splaiul \\ Independentei 313, Bucharest, Romania, adrian.petrescuecs.pub.ro
}

\begin{abstract}
New pressure factors are threatening the sustainability of the modern health systems. According to the European Commission assessments [1], the demographic changes, are changing the diseases patterns and, along with the bioterrorism and the major physical and biological hazards induced by the technological and economic growth is causing new transmissible disease patterns. A second major aspect is the population ageing - a phenomena which is affecting the developed countries. All those issues, catalyzed by the rapid development of new technologies into the fields of communication, micro and nano technologies, powerful computing capabilities at affordable prices etc. are revolutionizing the way of predict, prevent and treat illness and have triggered a major development of the telemedicine. This paper presents the developments on the telemedicine technology undertaken by UTI Group in partnership with the Faculty of Automatics and Computer Science from the "Politehnica" University of Bucharest, Romania. The partnership aim is to develop solutions to improve the access, efficiency, effectiveness, and quality of clinical and business processes utilized by healthcare and social care organizations, practitioners, patients, and consumers in an effort to improve the health status of patients. This paper is focused on the distributed system architecture, telemedicine system of system (SoS) emergent behavior and describes the main aspects of the distributed telemedicine systems efficiency evaluation.
\end{abstract}

\section{KEYWORDS}

Telemedicine, Distributed systems, Emergent behavior, Efficiency, Human factors

\section{INTRODUCTION}

Into the modern society, one of the biggest challenges refers to the demographic changes, including ageing and diseases pattern changing, which place a great pressure on the sustainability of the health systems. The technology developments generate new biological and physical security hazards; the bioterrorism became a major threat for the urban crowded areas; the climate changes are causing new patterns for diseases transmission. Based on the technology developments, the healthcare community is looking for new methods to meet these challenges. Additionally, due to the extension of the life span expectation and due to the lower births rates, the phenomena of population ageing become more and more effective with large implications into the cost of the healthcare and social assistance services costs, which have been increasing during the last decades. This trend will eventually lead to an increasing number of lonely elderly or chronic diseases people needing social assistance. At the same time, the hospital healthcare of those persons is likely to become financially not feasible. Thus, a more and more essential task for the today's societies is to improve the quality of life for an increasing fraction of elderly or disabled people. This phenomena becomes especially important into the industrialized countries confronted with this demographic shift. According to [1], at the end of the 19th century, life expectancy for males and females in Europe was 45.7 and 49.6 years, respectively while by the year 2000, this has increase a result, the EU population that is becoming increasingly older. 
Since the process of ageing is on an ascendant slope within the industrialized countries, this problem is aggravating. Based on the technology developments, the healthcare community is looking for new methods to meet these challenges and is seeking approaches where:

- Holistic approach: The patients are viewed in their totality: apart from their health status, the social network and their individual capabilities and preferences are also considered.

- Partnership: The clinicians and the patients are working as a team in order to achieve the best outcomes.

- Patients remain at home: The quality of life of any person, regardless the age, heavily depends on the efficiency, comfort and cosiness of the place named "home". For elderly people, home is a place of memories where they spend most of their time. Upon ageing, the patient demands on their home environment will increase, especially when their health status starts to get worse. Disabled people have specific requirements as for their home environment and its functionalities.

All those issues, catalyzed by the rapid development of new technologies into the fields of communication, micro and nano technologies, powerful computing capabilities at affordable prices etc. are revolutionizing the way of predict, prevent and treat illness and have triggered a major development of the telemedicine. The work described in this paper is based on the UTI large experience in $\mathrm{R} \& \mathrm{D}$ activities for system integration and product development for personnel, assets and vehicles monitoring, e.g. the KTrackP [2] used for remote monitoring for remote monitoring of the patients and personnel involved in special activities:

- Medical surveillance: collection of patient's the blood pulse and temperature information, together with the position (distance to, or estimated time to arrival at the care unit).

- Sportsman training: effort monitoring, position (for endurance sports).

- Expedition monitoring: position, vital parameters, SOS.

- Military and special forces training: real time positioning, operational tracking and simulations, blues force tracking.

- Border police and special transports security personnel tracking.

The current telemedicine platform, a result of the scientific partnership between UTI and UPB, responds to most actual requirements of the telemedicine and serves as a technological enabler to help elderly people to stay independent into their own houses and to allow those suffering from chronically diseases and those living in isolated sites to be helped.

\section{Distributed Systems For Domotic Surveillance. Applications FOR LONELY ELDERLY OR CHRONICALLY ILL.}

The main goal of the research activity described by this paper is the technology support for independent leaving for elderly people and for remote care for the chronically ill persons leaving into their own homes. The extended work deals with a system of systems, which includes different systems dedicated for independent operational goals (localization, vital signs monitoring etc.) and reunites the individual capabilities into a complex "meta-system" with superior functionalities and performances. 
International Journal of Computer Networks \& Communications (IJCNC) Vol.3, No.1, January 2011

\subsection{Chronic Disease Monitoring}

Modern reserches reveal the the chronic diseases contribute to up to 75 percent of medical care costs in the US [3]. Those costs are estimated on a wide range of health problems, including sleep disorders, asthma and diabetes. Most often, it is necessary a sort of medical monitoring, especially into the advanced stages of the disease. Since not all chronic disease monitoring is the same, we further refine the category as follows:

- Episodic patient monitoring is often utilized in non-critical patients to track specific indicators and identify the progress of the disease or recovery. Into this use case is treated the non-acute or episodic patient monitoring. Usually are monitored the patient's vital signs ( temperature, heart-rate etc) as well as disease specific indicators (ECG, blood glucose level, blood pressure etc). The main goal is to automatically detect anomalies and spot trends. This use case also covers periodic medical examinations involving wearing of a set of medical sensors; the readings are interpreted by the doctors and archived for later further analysis. All the information collected during the monitoring is time stamped and securely forwarded to a gateway that functions as a patient monitoring system. Additionally, the gateway forwards the aggregated information in a secure way to a database server. The medical personnel and the family can access the information stored in the database server to monitor the progress of the disease. The latency of transmitting the information to the gateway is not critical in this scenario since all the information is time-stamped and the patients are not in a critical state; the data can be stored locally at the medical device and/or gateway and securely transmitted only when a predetermined amount of data is gathered. It is also possible that the medical devices and/or the gateway perform some type of data compression to minimize bandwidth use.

- Acute conditions that require constant or frequent measurement of health status is often associated with the continuous patient monitoring in order to allow continuous measurement of patients' health status at rest or during mild exercise for purpose of treatment adjustment, recovery or diagnosis. The vital signs (e.g. heart rate, temperature, pulseoximeter) measurements waveforms ) are securely forwarded to an wearable data collection unit for sequential storage and/or data fusion. The acquired data is forwarded to a home/local off-body gateway; this device performs sensor configuration, storage and data analysis as wll as long range data communication. Another option is to send the data directly to a mobile terminal. The health care professional uses the captured data to provide the appropriate diagnosis or to adjust the treatment level.

- The system can also issue alarms based on preset condition for each specific patient and disease; therefore is mandatory to have a continuous monitoring of the vital signs. All collected data are time-stamped, locally stored by the acquisition platform and forwarded, via the local gateway, to the remotely located doctors (medical clinics, hospitals etc). Obviously, in this use case is necessary to achieve minimum bit error rate and maximum end-to-end latency not to exceed a few seconds. It is also possible to automatically issues response actions from the monitoring system, upon patient alarm processing. For instance, family members can be warned, security, safety and social care services may be alarmed. On the medical side, for example, if during the monitoring of a diabetic patient the blood glucose level falls below a certain threshold, an alert can be sent to the patient, physician(s) and/or medical personnel. 


\subsection{Patient state monitoring}

Apart from the health status monitoring, an important aspect is the activity and general status monitoring as well as patient safety monitoring, especially for the persons above 65 years. In this respect, some of the most important applications are elderly people monitoring and people safety monitoring.

\subsubsection{Elderly people monitoring scenario}

This use case focuses on monitoring an elderly person's daily activity. Therefore, it is necessary to monitor, beside the medical sensors/devices for the vital signs, additional non-medical sensors such as environmental sensors, motion sensors, home sensors (e.g. bed, door, window...), security sensors (broken glass, door open too long...) etc. Similarly with the previous use case, all the information is sent to the local gateway for processing, storage and long range communication with the caregiver and/or family members. All those may, upon permission, access the information and asses the status of the elderly people. If certain predetermined events occur, automatic responses could be triggered. Another important feature is monitoring the behavior pattern of the elderly people. For instance, if the elderly person has to follow a specific daily schedule such as reading the glucose level and weight measurement in the morning, the doctor can monitor all those actions and, is a certain routine is not respected, can be sent a reminder to that person and, if the person not responds to set number of reminders, the caregiver can be sent an alarm. Necessarily, in this use case, the acquisition platform and the local gateway must be able to handle both medical and non-medical information in both ways: from patient to doctor and vice versa. This use case also covers journaling, a technique that is recommended for patients to help their physicians diagnose certain conditions, such as rheumatic disease. All the information provided by both the patient and by the other medical or non-medical devices in the home network is recorded and stored in a central monitoring centre database server for later/further review by the doctors and/or family. Additionally, using this technique, it is possible to aggregate patient records and medication, environmental or behavioral changes and correlate them with body function changes (e.g. fatigue, motion range, etc.) and, thus, to enable preventive actions.

\subsubsection{Safety monitoring scenario}

The safety monitoring scenario deals with the home safety (sometime with the home security). In this respect, the home environment is monitored in order to detect, in an as early as possible stage, toxic gases, water floods and fire. Additionally, the vital signs (e.g. heart beat, temperature) of the persons in the home are also monitored. The data gathered by the medical and non-medical devices is analyzed locally and/or securely forwarded to a gateway for processing and storage. If predetermined events occur, the caregiver and/or family receive alerts. Automatic responses can be triggered when certain events occur.

\section{SYSTEM ARCHITECTURE}

Healthcare and social care organizations include a wide range of professional, operational and functional groups, distributed on large geographical areas. Hence, the stakeholders structure is quite diverse since it includes medical and social workers, healthcare decision makers, patients etc. Thus, due to the large scale and distributed architecture, a system of systems (SoS) approach was adopted for an evolutionary development of the components: patient monitoring, hospital care, ambulance service guidance to intervention and on route patient assistance, first responders emergency management. The system of systems topology is depicted into the next figure. 
At the home level (although this term is merely generic since we can discuss also about the monitoring patients in remote locations, in ambulances being in traffic etc...), the system is monitoring the patient's vital signals, as well as data related to the daily activities patterns (where applicable). This framework is applicable on both home and assisted care facilities monitoring.

The main functions of the system are:

- Monitoring of the chronically ill patients living into their own homes.

- Elderly people monitoring.

- Social assisted people monitoring.

- In-transit monitoring of the patients transported by the ambulances.

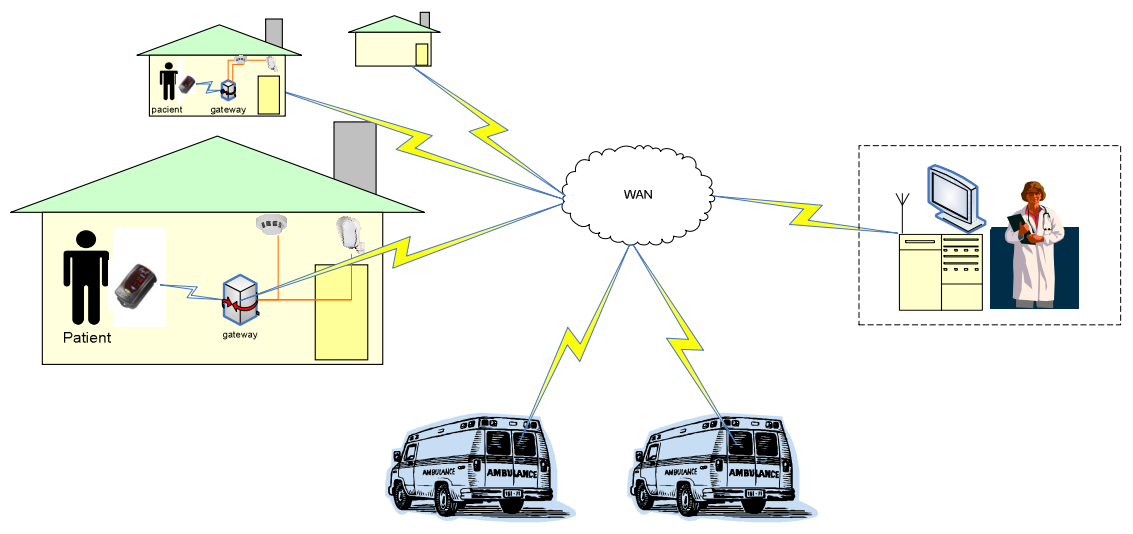

Figure 1. System Architecture

The main components of the system are (see next figure):

- A dispatch centre, having the following main functions: sensors constellation management, data processing and storage, source of information foe the medical staff, patients and family members (using secured data connection).

- Personnel data collection systems.

- Patient and social assisted people home monitoring.

- Patients in-transit visibility system.

- First responders assistance system.

- Software applications package, customized for each stakeholder category

The main data category acquired by the system are:

- Medical data : ECG, blood pulse, blood glucose level, blood oxygen level, body temperature.

- Patient's activity and general status data: position (including indoor positioning), fall detection, weight.

- Safety and environmental data: ambient temperature, fire alarm, toxic gases detection. 


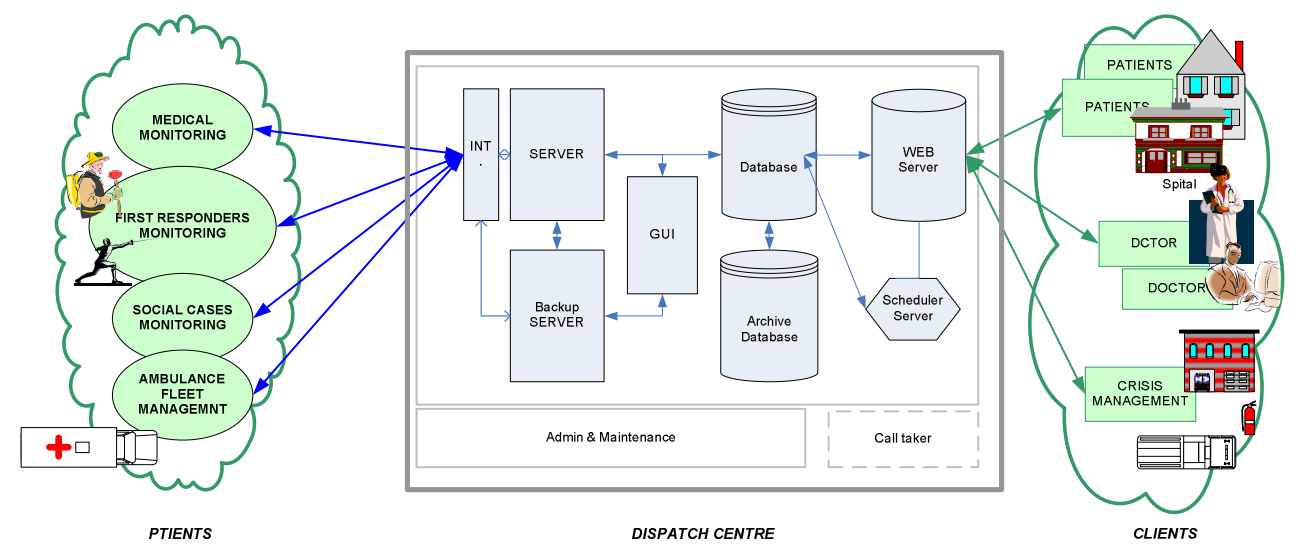

Figure 2 System components

All the technical capabilities of the system must eventually support the remote medical monitoring, the purpose itself of the system. Several factors play a role in specifying the functionality and constraints of our system. Here we list the system design points.

- Wearability and usability. In order for a system to be successful users must be willing and able to use it. Therefore, the sensor must conform to a reasonable form factor and require as little user intervention as possible to use it.

- Long lifetime. The sensor and mobile device must conserve energy to extend the life of the battery for as long as possible. Reducing communication costs and active computation serve to meet this objective.

- Accuracy. The signal processing on board the sensor must be accurate and resilient to many types of signals. No body sensor will be able to sense the 'perfect' waveform because of changes in the electrode-skin interface, motion artifacts, and quantization errors, for example. Real-world difficulties inherent in sensing data must be overcome to reduce false positives and false negatives.

- Near real-time. Sufficient performance dictates that the sensor data are processed and propagated through the system with reasonable delays (which will be inevitable due to the routing of the sensor data from the sensor through a mobile device to the Internet).

- Conformity to security best practices. A design that treats security as an afterthought or ignores it altogether is inappropriate in today's society. Basic encryption, authentication, and authorization protocols should be utilized throughout the system.

\subsection{In-house patient monitoring system}

This system used to monitor the patients living into their own homes (applicable for both chronically ill patients and elderly people living alone) has the following structure:

- Central monitoring unit (UCM - CMU);

- Wearable sensors platform (PSP - WSP);

- $\quad$ Stationary sensors platform (PSF - SSP); 
- Environment data acquisition unit (PM - EDC);

- Mobile units (TM - MU).

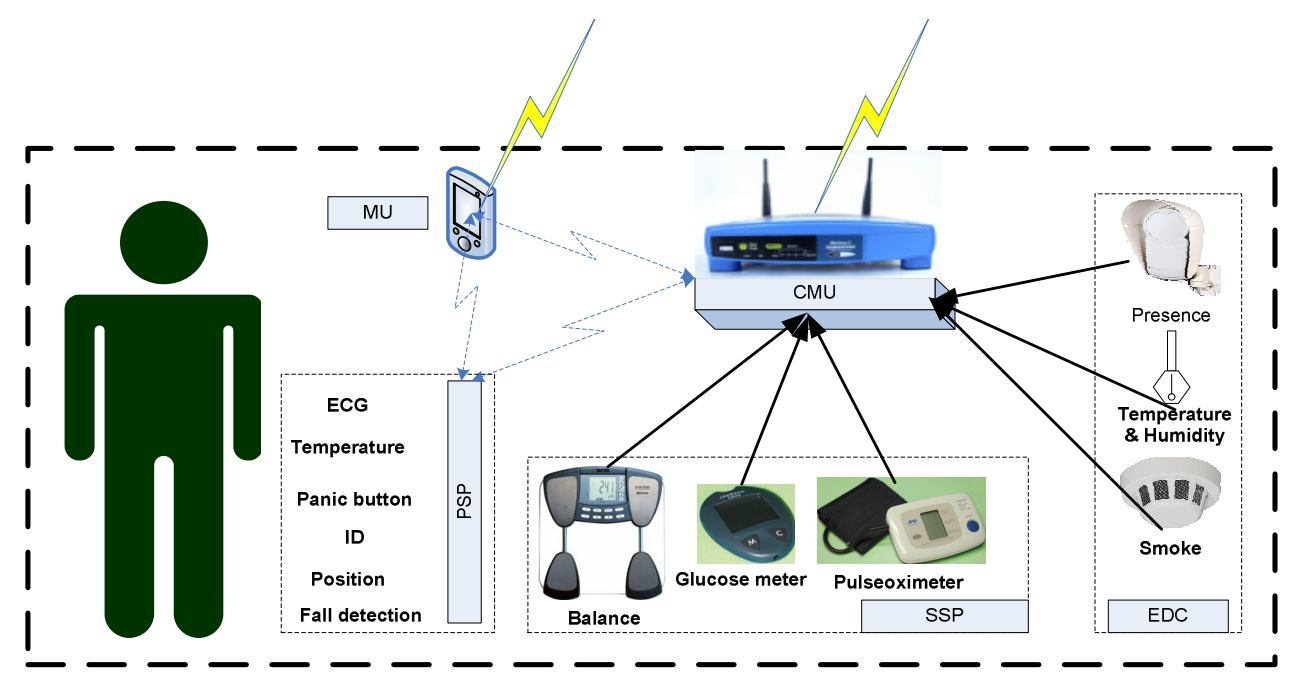

Figure 3 Patient's home monitoring system

\subsubsection{The wearable sensors platform}

The wearable sensors platform is collecting the patient data and sends them wirelessly to the Central Monitoring Unit. Upon pressing the panic button or upon automatic fall detection the wearable sensors platform issues an alarm and sends it to the monitoring centre, via the central monitoring unit or via one of the mobile terminals. The platform may be equipped with a GPS receiver for outdoor localization. For indoor localization are used RFID tags placed in relevant locations inside the house. The system uses passive tags as well as low-power active tags. All those tags are sensed by an RFID reader embedded on the platform; by associating the tag ID with is pre-assigned position it is detected the platform's location inside the house.

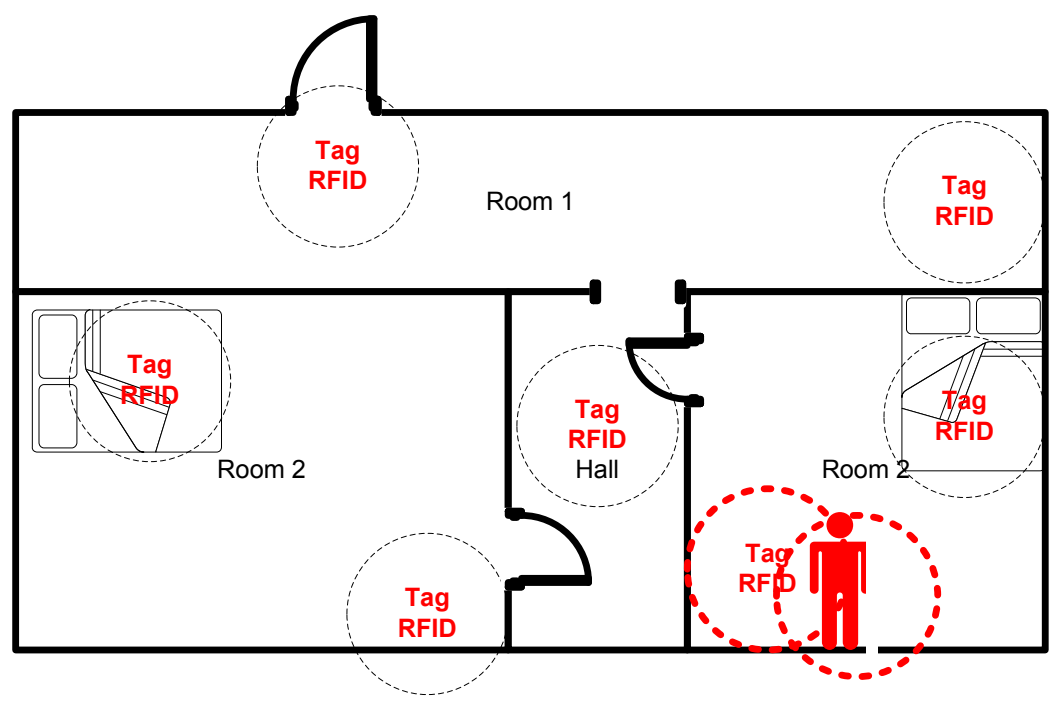

Figure 4 Indoor localization 
The wireless sensors platform architecture is presented into the next figure.

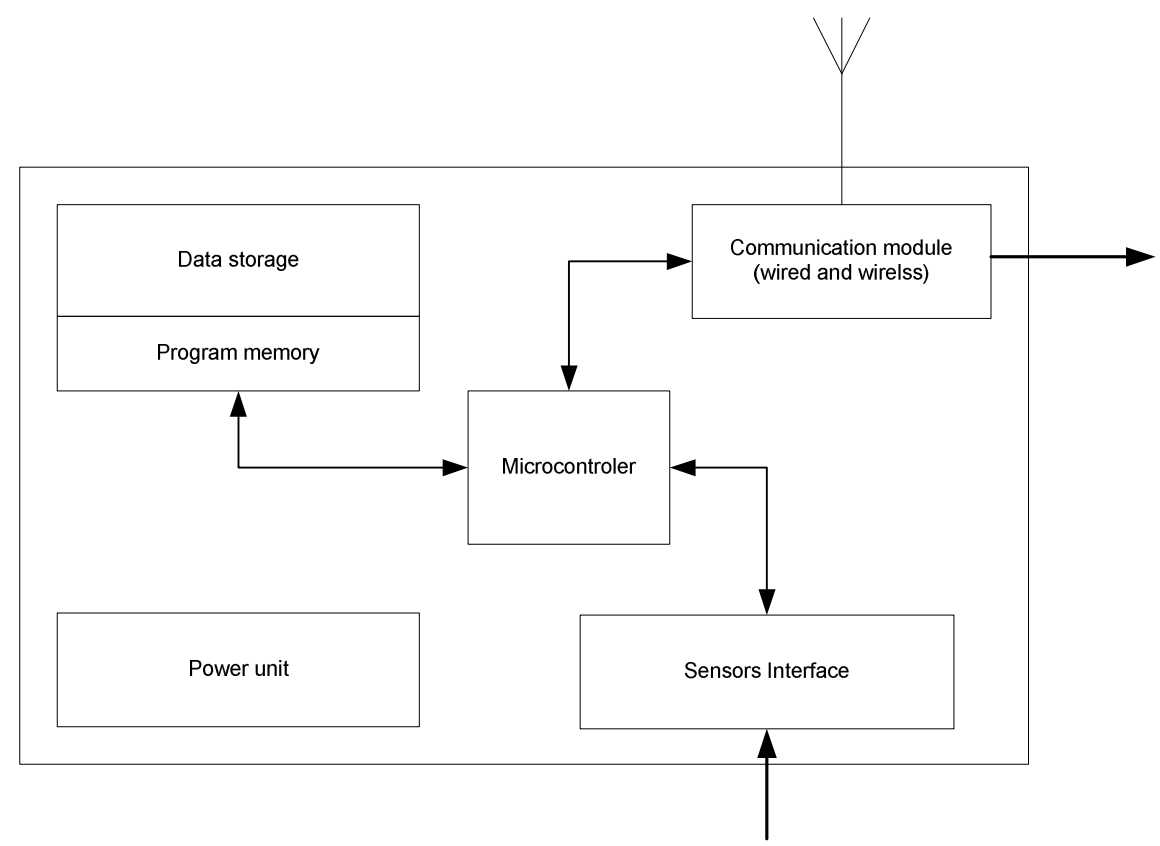

Figure 5 Wearable monitoring platform

The platform main features are:

- importance: mandatory;

- aquired data: ECG, ambient temperature, body temperature, fall detection, panic button, position;

- used both indoor (room level) and outdoor (5-10 meters accuracy);

- frequency and duration of use: continuously during the monitoring;

- low power wireless communication with the central monitoring unit and the mobile terminals.

- Design constraints: low size, light weight, low power consumption, co-existence with other wireless communications (such as Bluetooth).

\subsubsection{Stationary sensors platform}

Actually, the stationary sensors platform is a set of data acquisition devices, used to collect patient's health status data upon request. The main platform features are:

- importance: optional, dependent by the patient monitoring scenario;

- aquired data: body weight, blood oxygen level, blood glucose level;

- used only indoor; 
- frequency and duration of use: upon request;

- low power wireless communication with the central monitoring unit and the mobile terminals;

- Design constraints: low size, light weight, low power consumtion, co-existance with other wireless communications (such as Bluetooth).

\subsubsection{Environment data acquisition platform}

The Environment data acquisition platform is a device used to collect the data related to patient's environment. This device is acting as a "home controller" and, besides sending the data to the main monitoring unit; it may also work as an independent domotic system. Generally, this module is collecting the data related to the home indoor temperature and humidity, fire detection as well as presence detection.

The main platform features are:

- importance: optional, but it is strongly recommended in order to complete the information with the environment data;

- acquired data: temperature and humidity, smoke and fire detection, presence detection;

- used only indoor;

- frequency and duration of use: continuously during the monitoring;

- low power wireless communication with the central monitoring unit and the mobile terminals;

- Design constraints: power autonomy for at least 72 hours (in order to be compliant with the national regulations), co-existence with other wireless communications (such as Bluetooth).

\subsubsection{Mobile Terminals}

Those terminals are smart-phones or PDAs, used in order to display information and/or to alarm de patient. The mobile terminals allow the patient to visualize information related to the health status, medications and to communicate with monitoring centre and/or the family.

\subsubsection{Central monitoring unit}

The Central Monitoring Unit is acting as a local gateway, receiving the information from the sensors platforms. This unit is also performing data aggregation and local processing and storage as well as long range communications via GPRS channels. Additionally those modules are allowing "hands-free" communication channels with the monitoring centre. The central monitoring unit is polling periodically (according with a predefined schedule) the sensors platforms.

The central monitoring unit has also additional functions, such as verbal memos used to remember the patient to follow the medication timely, such reducing the risk of the accidentally 
International Journal of Computer Networks \& Communications (IJCNC) Vol.3, No.1, January 2011

overdose or missing the regular dose. In the same way, it is possible to send memos related to scheduled health status check, and thus achieving an appropriate treatment program.

The module's main features are:

- importance: mandatory;

- used only indoor;

- frequency and duration of use: continuously during the monitoring;

- low power wireless communication with the sensors platforms and the mobile terminals;

- Design constraints: low power consumption, power autonomy for at least 72 hours (in order to be compliant with the national regulations), co-existence with other wireless communications (such as Bluetooth).

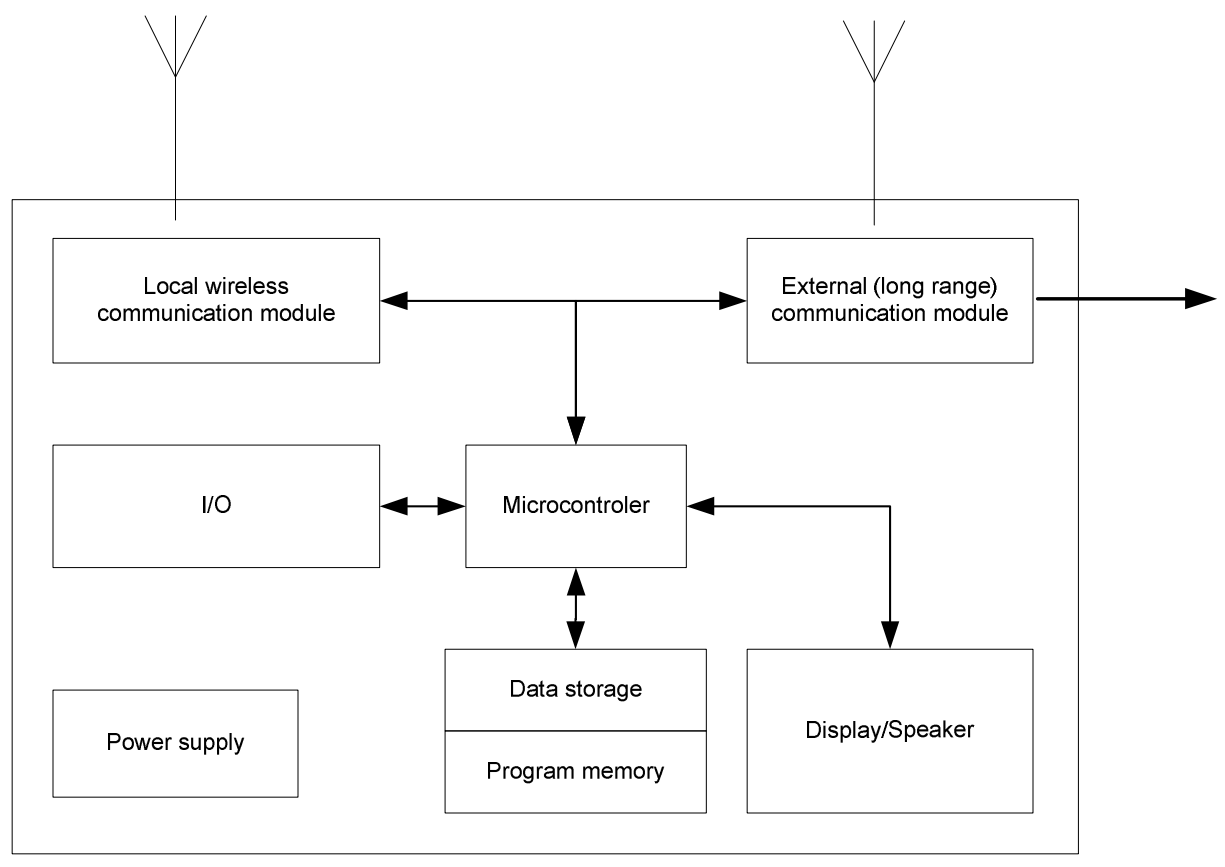

Figure 6 The Central Monitoring Unit architecture

\subsubsection{In-transit patient monitoring unit}

The In-transit monitoring unit is combining the information related to the patient health status with the ambulance data (position, speed etc); thus, it is extending the patient monitoring during the transportation to/between the hospital/s. The patient health status information is presented into the next figure.

The patient data is combined with the vehicle data and sent to the monitoring centre; the consolidated data is contributing to a better patient treatment (for instance, a specific parameter 
related to a vital sign monitoring may be combined with the estimated time to arrival to the hospital based to the current position and speed, street availability etc ).

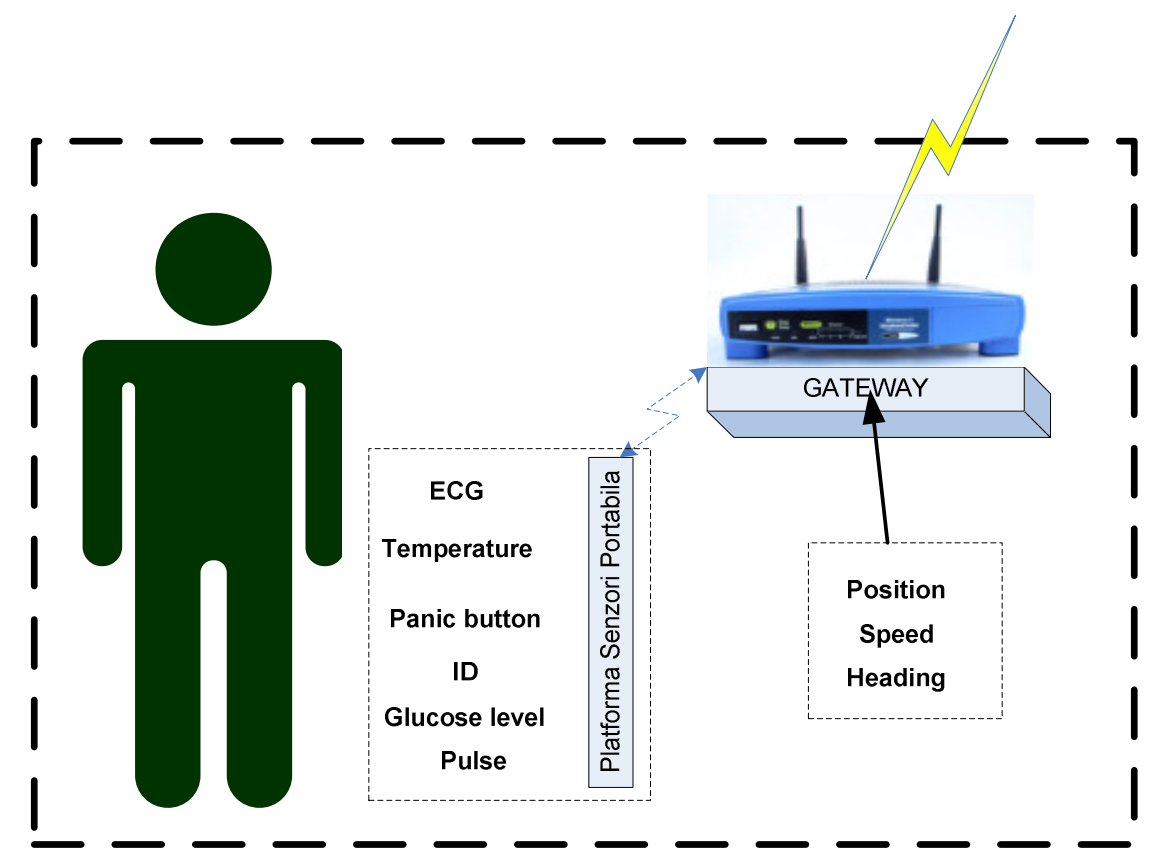

Figure 7 In-transit patient monitoring unit

The module's main features are:

- importance: optional;

- used outdoor and ambulance onboard;

- frequency and duration of use: during the transportation;

- long range wireless communication with the hospitals;

- Design constraints: vehicle onboard installation, low size, low power consumption.

\subsection{The integration strategy}

Apart from the physical integration (obviously necessary since integration of different equipments with specific communication protocols is required), a functional integration is necessary, in order to overcome the fuzzy functional partition among different systems, a source of potential functional conflicts. The information offered for the medical and social assistance staffs has also to pass a semantic integration since this information is used by different actors with different needs and perspectives over the same subject: the patient status. In this respect, the monitoring centre system is acting as a system-of-systems bridge, being responsible for the physical, functional and semantic integration aspects. 


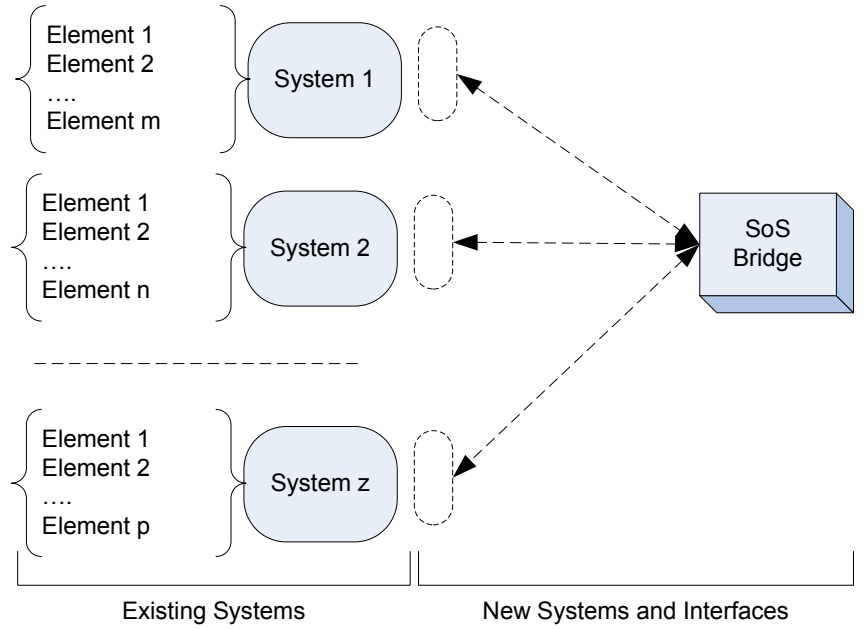

Figure 8 SoS Bridging

\section{Data Management}

Generally, a system-of-systems is including a large number of component systems, being necessary a data architecture compliant with two major requirements:

- although autonomous systems are integrated, it is necessary to preserve the semantic and the consistency of the data handled and stored;

- it is necessary a persistent storage of the data handled inside the system-of-systems although an information is belonging with a single system, it may be used by several others inside the same system-of-systems.

The easiest method is to store the data in a centralized manner inside the same system-ofsystems. This method implies the simplest architecture and, often, the lowest risks related to the stored data management. But it has two major disadvantages: it alters the component systems autonomy and, secondly, it is difficult to achieve an acceptable level of availability of the stored data. In order to deal with the high risk of heterogeneous data structure and semantic problems, a federated data management model [4] is to be used (Figure 9). The overall system-of-systems data repository contains shared data owned by the partner systems and posted to the repository into a predefined format.

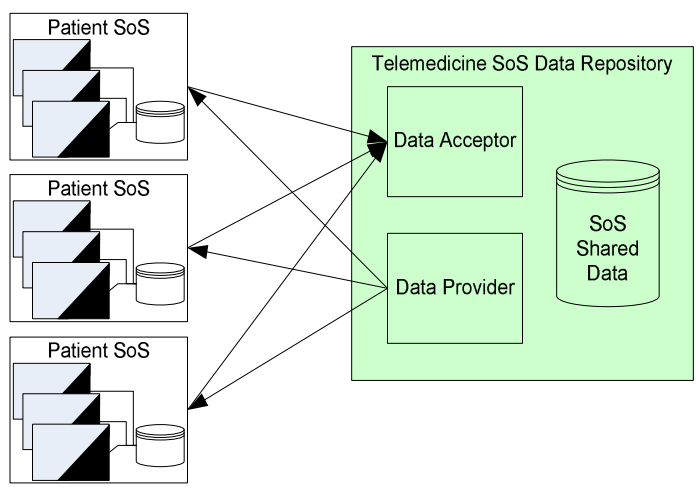


Figure 9. Data management

An important aspect in this architecture is the data protection, considering the need for interactions between the systems and/or users and the privacy requirements. In order to achieve an appropriate level of data security, the following aspects are considered [5]:

- Non repudiation: Identities guarantee for all the users accessing or providing resources.

- Confidentiality: Controlling the access to the information stored or manipulated into the system.

- Authentication: Identification methods for the personnel accessing the information.

- Integrity: Prevention of the unauthorized access to the information.

In a system of systems approach, data integrity is even more important as unintentional interference between the data holders may occur.

\section{TELEMEdicine SySTEM OF SySTEMS EMERGENT BEHAVIOUR}

One of the most important features of the system-of-systems is their emergent behavior: the overall heterogeneous system of autonomous systems has an emergent behavior which, with a heuristic management, is generating synergies among its components for the benefit of a much wider social scale. As a consequence, by strategic connections with other applications for human life protection (security, emergency and disaster management services, etc.) the quality of the medical and social services is improved as well as the social awareness and preparedness for disaster management.

The term "emergence" was used for the first time by the British thinker GH Lewes, more than 100 years ago. The emergent behavior became one of the most important features of the complex systems. Examples of emergence may be found both in nature and into the human behavior:

- Individual ants don't know about the coordinated search for food; the whole group knows.

- The stock market is an example of emergent systems at a large scale.

- The traffic slowdown upon tunnel entrance approach, although there are no speed limit signs, is another example.

In system-of-systems the emergence may bring additional unique features, whenever the new features are not conflicting with the overall goal. There is no generally accepted definition for the "good" or "bad" emergence but a feasible way to evaluate it is to monitor the effects of the new features over the overall system-of-systems.

Another important aspect of the system-of-systems emergent behavior is the impact over the overall system functional predictability. Naturally, the new emergent features of the system-ofsystems lead to new features not foreseen at the design stage; therefore, those new features make impossible to predict the system evolution, at a large time scale at least.

A large set of events are generated whenever an incident threatening the patient life or safety occurs; this often involves a wide span of stakeholders such as: family, medical care, social services etc... That's the moment when the emergent behavior of the telemedicine systems of 
systems is more visible due to the weak bondage between the component systems. Although hierarchically structured (see Fig 10), the component systems are operationally and managerially independent so the hierarchy is not closed, allowing dynamic adaptation to the operational conditions and fostering the self-organization and individual specialization of its components for the benefit of the entire system of systems.

Self-organization is a bottom - up process, in which the internal organization of a component system, most likely an open system, increases in complexity without being guided or managed by an outside source. This eventually leads to the need of flexible but effective system governance, needed in order to achieve an appropriate level of efficiency.

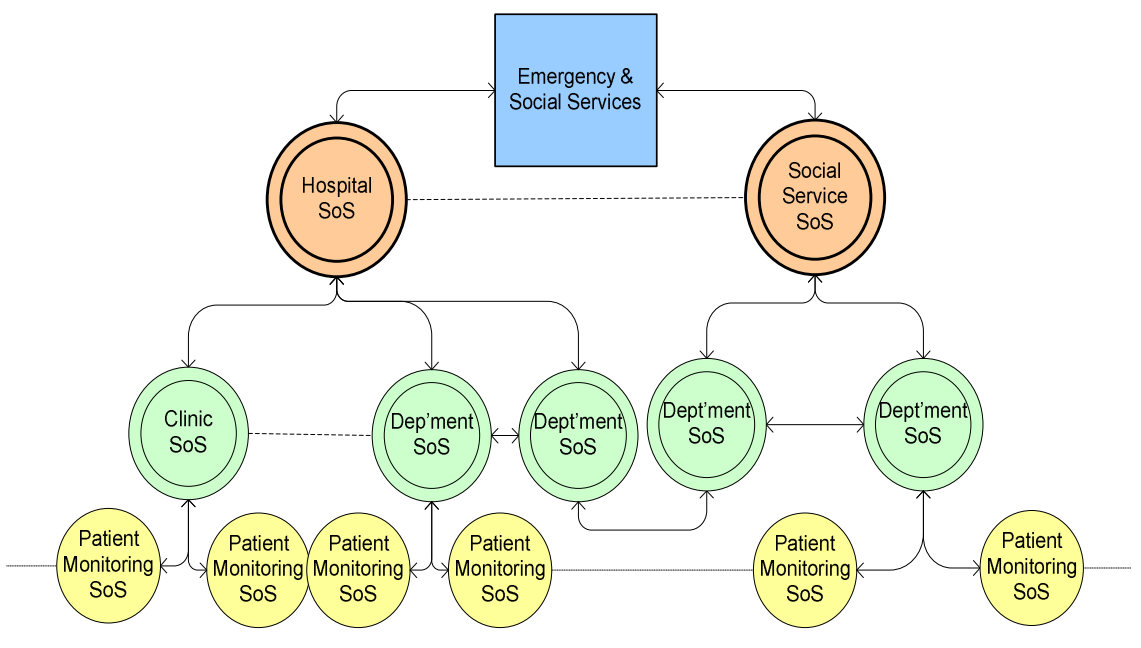

Figure 10. Telemedicine system hierarchy

\section{TELEMEdicine SySTEM OF SySTEMS EFFICIENCY}

An important aspect of the telemedicine endeavors is the efficiency, especially into a classical approach dominated environment: telemedicine versus available hospital \& clinical care. That's because, changes in clinical outcomes can take years to identify, and causes are often difficult to isolate. In the meantime, health managers are striving to identify and tailor programs that are delivering the expected values or modifying the aspects that are not working. Therefore, it is difficult to measure the value generated by the technology-based systems versus the results achieved by classical medical systems.

Albeit technology investments, accompanied by careful planning, training, post-commissioning support can improve the delivery of healthcare services and enable far-reaching social and economic value, the "traditional" healthcare value categories are still applicable to the telemedicine systems. Among them, we can identify the following main healthcare value categories and possible key performance indicators (KPIs) that may be used to measure them.

Table 1. Healthcare value categories and associated KPIs

\begin{tabular}{|l|l|l|}
\hline $\begin{array}{l}\text { Value } \\
\text { Category }\end{array}$ & Description & Possible KPIs \\
\hline Medical & This is the cost of delivering a clinical & - Reduction in acute care costs. \\
service & service which may be slashed either by & - Percentage reduction in cost of \\
cost & more timely intervention (reducing the \\
reduction & managing patient case. \\
overall cost per patient) or by reducing & - Quicker recoveries requiring less \\
\hline
\end{tabular}


International Journal of Computer Networks \& Communications (IJCNC) Vol.3, No.1, January 2011

\begin{tabular}{|c|c|c|}
\hline & $\begin{array}{l}\text { the overall cost of monitoring patients } \\
\text { (through reduced travel and associated } \\
\text { costs). }\end{array}$ & resources. \\
\hline $\begin{array}{l}\text { Patient } \\
\text { Safety }\end{array}$ & $\begin{array}{l}\text { By telemedicine collaborative platforms, } \\
\text { doctors from different locations, with } \\
\text { different skills and levels of } \\
\text { competences can work together, leading } \\
\text { to an improved decision making act, } \\
\text { resulting an improvement in patient } \\
\text { safety. }\end{array}$ & $\begin{array}{l}\text { - Percentage increase in collaboration } \\
\text { between clinicians and healthcare } \\
\text { workers in the community. } \\
\text { - Percentage increase in early } \\
\text { identification of worsening of patient } \\
\text { conditions. }\end{array}$ \\
\hline $\begin{array}{l}\text { Patient } \\
\text { Satisfacti } \\
\text { on }\end{array}$ & $\begin{array}{l}\text { Provide relevant and timely diagnosis } \\
\text { and monitoring to patients that is } \\
\text { convenient and non-intrusive to them. }\end{array}$ & $\begin{array}{l}\text { - Reduction in travel to hospitals and } \\
\text { clinics } \\
\text { - Reduced anxiety and increased } \\
\text { confidence/ empowerment } \\
\text { - Percentage adoption level among } \\
\text { potential patients } \\
\text { - Improved clinician communications }\end{array}$ \\
\hline $\begin{array}{l}\text { Quality of } \\
\text { Care }\end{array}$ & $\begin{array}{l}\text { A comparable or improved level in the } \\
\text { quality of care provided to patients } \\
\text { enabled through timely intervention by } \\
\text { GPs based on monitoring and evidence } \\
\text { based medicine }\end{array}$ & $\begin{array}{l}\text { - Proactive and timely intervention } \\
\text { by medical staff based on remote } \\
\text { monitoring centers. } \\
\text { - Percentage reduction in health crisis } \\
\text { due to proactive monitoring of } \\
\text { trends. } \\
\text { - Quicker access to care and } \\
\text { diagnosis. }\end{array}$ \\
\hline
\end{tabular}

Apart of these values categories, we can identify additional value categories specifically relevant to telemedicine solutions. These categories are transversal to healthcare value categories and offer a broader perspective, and eventually enhance or impede the success of telemedicine initiatives. The values considered as most relevant by us are:

Accessibility: Technology investments can increase citizen access to services by improving health worker productivity. Technology solutions that improve efficiency and reduce demands on secondary care can help control rising costs, leading to a broader patient base. The KPIs associated with this value category are:

- Percentage of population with access to telemedicine equipment.

- Percentage of services that are supported by telemedicine solutions.

- Availability of telemedicine solutions in public places.

Education: Due to the shift into the medical act approach, from the face-to-face discussion with the physician, to remotely operated evaluations or technology based automatic monitoring, it is necessary to provide education plans that educate patients on topics such as pain management so that they can better understand their condition and how to manage it review of their treatment history and plan. The KPIs associated with this value category are:

- Percentage of patients with access to online educational materials and effectively using these materials. 
- Percentage of patients that are provided with training via videoconferencing.

- Percentage reduction in the volume of general or routine queries to physicians.

- Percentage of patients who understand their symptoms and who can self-administer.

- Access to clinical results online.

Policy and Public Health: Strategic health decision makers or advisors can set policy and standards and define macro measures within a region that is aligned with the expectations of central government and supports increased institutional integration and improved health outcomes. The KPIs associated with this value category are:

- Percentage increase in access to healthcare services in rural communities

- Greater integration of care across institutional boundaries

- Percentage of healthcare services that are supported by telemedicine.

Overall, we consider that a successful telemedicine solution requires not only technology but also social service and healthcare partners involvement, in order to mix their expertise together with defined risks and benefits.

\section{HUMAN FACTORS APPROACH}

Upon ageing and illness progression, the patient is expected to experience decrements in hearing, vision manual dexterity, strength and memory. Therefore, special challenges are posing the experience and work of the designers of such systems [6].

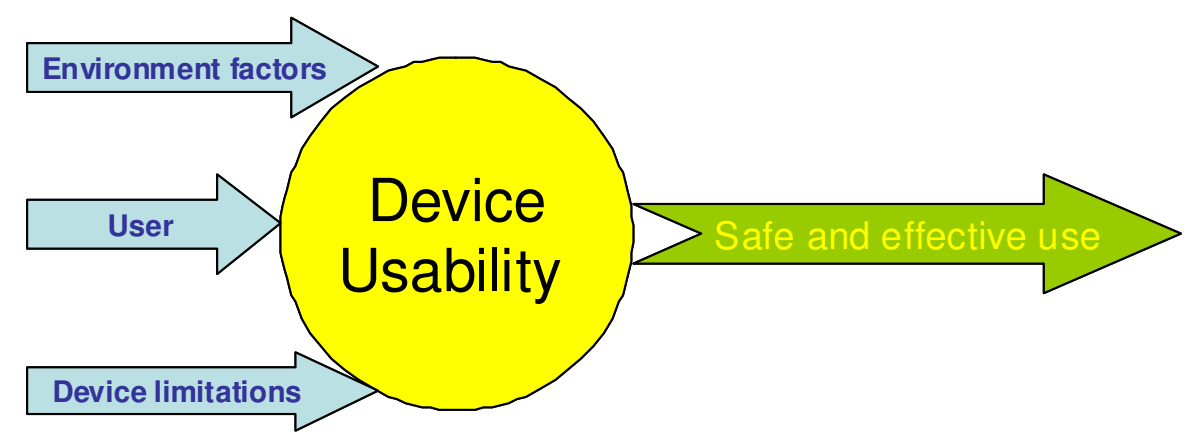

Figure 11. Telemedicine system hierarchy

Among the methods used to assess the needs of this particular population are interviews, surveys, focus groups and experimental research. The results proved that an integrated monitoring system can both help the person who's using it by providing around the clock assistance, and lifts an evermore heavy burden off the medical care system.

\section{CONClusions}

The telemedicine systems of systems approach is a way to enable extremely powerful strategies for preventing diseases and improving health and the overall quality of life for elderly and chronically ill persons living alone into their homes. When taken one by one, the architecture of 
each system component might appear simple, but the overall system eventually leads to a very complex system of systems. According to the individual characteristics of the systems, important heterogeneous information and emergent developments are extracted. The amount of the sensed data is very vast and complex, due to the large number and types of sensors which are continuously monitored in order to collect data over long periods of time. All those multiple entities have different goals, making the integration and system governance a critical issue. Success in telemedicine system of systems requires recognition, effective management, and exploitation of emergence.

\section{ACKNOWLEDGEMENTS}

The authors would like to thank for the support offered by UTI and the Faculty of Automatics - Politehnica University of Bucharest.

\section{REFERENCES}

[1] Assessment of the Senior Market for ICT. "Seniorwatch 2006 - Progress and Developments", European Commission, Information Society and Media Directorate General

[2] V. Petcu, "Advanced Security Technologies for Remote Surveillance In Distributed Systems," Proceedings of IntelliSec 09 - The 1st International Workshop on Intelligent Security Systems 11-24th November 2009, Bucharest, Romania, pp. 199-204, 2009

[3] ZigBee Wireless Sensor Applications for Health, Wellness and Fitness, 2009, ZigBee Alliance

[4] R. Cole, "Systems of Systems Architecture", in Systems of Systems Engineering - Principles and Applications, CRC Press, 2009, pp.37-70

[5] Warkentin, M. and Vaughn, R. "Enterprise Information Systems Assurance and Systems Security. Managerial and Technical Issues”. IGI Publishing, Hershey, PA, 2006

[6] D.W. Repperger, "Human Factors In Medical Devices", in Encyclopedia of Medical Devices and Instrumentation, Second Edition, J. Wiley \& Sons, Inc. 2006, pp. 536-547

\section{Authors}

Viorel Petcu is the Chief Technology Officer of UTI Security and Defense Division. He has graduated the Faculty of Electronics and Telecommunication in 1993; he is PhD student at the Faculty of Automation and Computers, Politehnica University of Bucharest. He has been leading research activities on both Romanian and European RTD programs; his main research interest includes telemedicine and security systems.

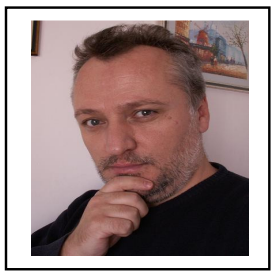

Adrian Petrescu is a professor at the Faculty of Automation and Computers Science, Politehnica University of Bucharest, and a member of the Academy of Technical Sciences from Romania. His research interest includes the reconfigurable digital systems design, the VLSI design and telemedicine. He received a $\mathrm{PhD}$ in Computer Science from the Power Institute-Moscow, in 1964.

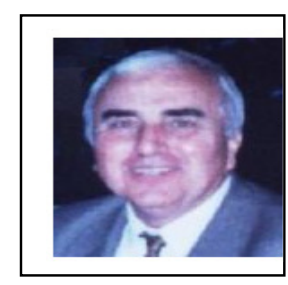

